\title{
Controlled side-by-side assembly of gold nanorods and dye molecules into polymer-wrapped SERRS-active clusters ${ }^{\dagger}$
}

\author{
Alison McLintock, Nathan Hunt and Alastair W. Wark* \\ Received (in $X X X, X X X)$ Xth $X X X X X X X X X 200 X$, Accepted Xth $X X X X X X X X X 200 X$ \\ ${ }_{5}$ First published on the web Xth $X X X X X X X X X 200 X$ \\ DOI: 10.1039/b000000x
}

\begin{abstract}
The controlled side-by-side assembly of gold nanorods in solution together with Raman reporter dye molecules to create small SERRS-active clusters stabilised by a surrounding polymer layer 10 is demonstrated. This promising new class of nanotags offers several advantages over spherical nanoparticles for bioimaging and are of potential importance for a wide range of plasmonenhanced spectroscopies and can also serve as building blocks for more complex solution-phase nanostructures.
\end{abstract}

${ }_{15}$ Gold nanorods have recently emerged as an attractive alternative to spherical metallic nanoparticles for exploring a wide range of surface plasmon enhanced sensing and biomedical applications. ${ }^{1-3}$ By adjusting the rod aspect ratio it is possible to achieve a strong optical extinction at targeted 20 wavelengths varying across most of the visible and nearinfrared (NIR) regions of the electromagnetic spectrum. Also, the geometrical and surface chemical anisotropy of the rodshape offers potential avenues for assembling more complex nanostructures. Nanoparticle aggregation in the presence of a 25 specific reporter or target molecule is associated with dramatically enhancing the molecular detection signal of a variety of optical techniques, including surface-enhanced (resonance) Raman spectroscopy $(\mathrm{SER}(\mathrm{R}) \mathrm{S}) .{ }^{4}$ However, controlled assembly within colloidal solutions to create 30 stablised free-standing clusters $\sim 2-4$ particles in size is challenging for spherical particles with the additional engineering required to also incorporate Raman-active molecules within interparticle junctions an added difficulty.

In this communication, we show that gold nanorods can be 35 assembled in a side-by-side fashion with a high degree of uniformity to produce stabilised clusters predominatly composed of dimers and trimers. This is also achieved in the presence of a Raman reporter to create SERRS nanotags optimised for use in the NIR. There are only a few examples 40 in the literature of side-by-side rod assembly; ${ }^{5-8}$ all of which involve the uncontrolled formation of large clusters. In the case here, the collective resonance of the nanorod assembly can be tuned depending on both the rod size and the number of rods forming a cluster. This is a significant advantage 45 compared to spheres as a much larger range of resonance wavelengths can be easily targeted. Also, the maximum diameter of the rod cluster is kept close to that of the average length of a single rod $(\sim 50 \mathrm{~nm})$ while for comparably sized spheres, the overall aggregate size will be much larger and 50 more variable on dimer/trimer formation. ${ }^{9-12}$

An outline of the strategy for the preparation of the SERRS nanotags is described in Scheme 1. First, a mixed layer of cetyltrimethylammonium bromide (CTAB) and 3,3'diethylthiatricarbocyanine iodide (DTTCI) is assembled onto 55 the positively charged nanorod surface (step 1 in the scheme). The CTAB surfactant bilayer is responsible for both directing the rod shape during synthesis and maintaining colloidal stability. However, the presence of this bilayer also complicates subsequent surface functionalisation and this has

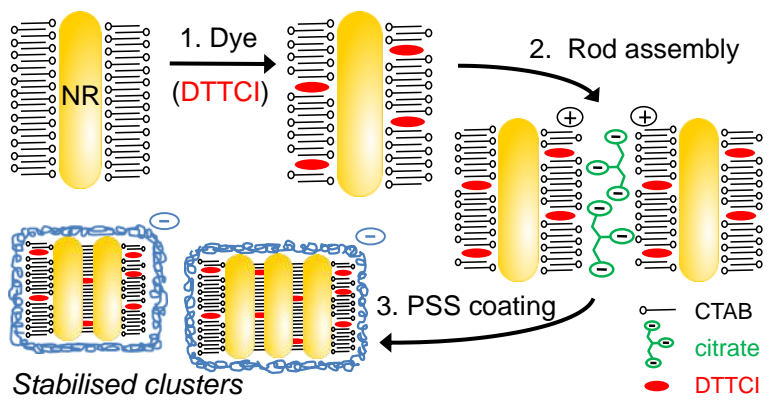

60

Scheme 1 Schematic outlining the preparation and stabilisation of selfassembled gold nanorod/dye clusters.

hindered the use of nanorods for SERS-based applications. Thus, instead of replacing CTAB, we instead selected a ${ }_{65}$ reporter dye, DTTCI, which was found to partition into the CTAB bilayer with a very high efficiency. Next, the side-byside electrostatic assembly of nanorods is controlled via the addition of citrate anions (step 2). Finally, in step 3, the clusters are wrapped with a polyelectrolyte layer of poly70 (sodium 4-styrenesulfonate), PSS, to stabilise the assembly.

Prior to nanotag formation, nanorod solutions were prepared using a modified version of the procedures developed by Murphy ${ }^{13}$ and El-Sayed ${ }^{14}$ with the reaction volume scaled up to $1 \mathrm{~L}$ (see $\mathrm{ESI}^{\dagger}$ ). All the experiments 75 described here were performed using the same stock solution with a particle concentration of $\sim 1 \mathrm{nM}$, an average rod length of $48 \mathrm{~nm}$ and 4.4 aspect ratio, and transverse and longitudinal plasmon resonance peaks at $511 \mathrm{~nm}$ and $800 \mathrm{~nm}$, respectively.

The choice of 3,3'-diethylthiatricarbocyanine iodide 80 (DTTCI) as a Raman reporter molecule was also based on it being strongly absorbing in the NIR $\left(\lambda_{\max }=765 \mathrm{~nm}\right)$ close to the laser excitation frequency of $785 \mathrm{~nm}$. In addition, due to DTTCI containing a quaternary ammonium functional group (Fig. S1, ESI ${ }^{\dagger}$ ) and also having poor water solubility led us to 85 surmise that the dye would have a high affinity for the CTAB bilayer coating on the nanorod surface. The rapid formation of a mixed CTAB/DTTCI layer was confirmed using fluorescence spectroscopy (see Fig. S2, ESI ${ }^{\dagger}$ ) which showed that in conditions where the dye molecule to nanorod ratio is 
1000: 1 and the bulk CTAB concentration fixed at $1 \mathrm{mM}$, the DTTCI fluorescence signal decreased by $>80 \%$ in around 1 min and was almost completely quenched 2 hours after introducing dye to a nanorod solution.

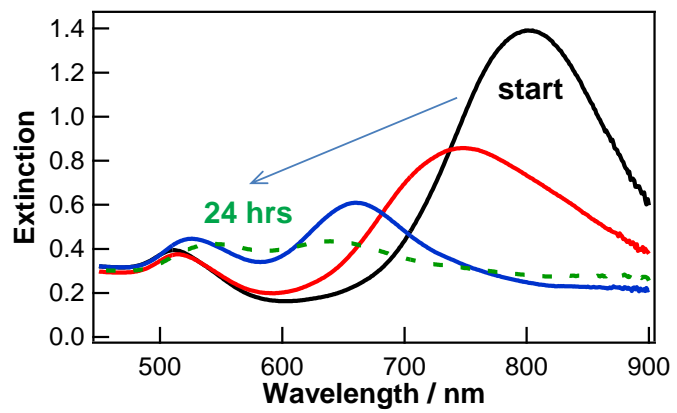

Fig. 1 Spectra monitoring the controlled side-by-side assembly of gold nanorods following the addition of citrate anions to a nanorod solution.

The resulting plasmon resonance shifts were measured over $24 \mathrm{hrs}$.

Fig. 1 demonstrates the control that can be achieved over 10 the side-by-side assembly of nanorods. Upon the initial addition of citrate ions to the rod stock solution (see $\operatorname{ESI}^{\dagger}$ ), the peak position of the longtitudinal plasmon resonance band gradually blue-shifts from a starting position of $802 \mathrm{~nm}$ to 641 $\mathrm{nm}$ as well as dampens over a period of 24 hours while the 15 transverse peak position correspondingly red-shifts from 511 $\mathrm{nm}$ to $545 \mathrm{~nm}$. The timescale and the extent of the rod assembly are dependent upon the ratio of citrate molecules to nanorods. At lower ratios the aggregation process is selflimiting with a fixed blue-shift obtained after a period of time, 20 while at much higher citrate concentrations the plasmon resonance is quickly damped due to random aggregates forming. Furthermore, when testing conventional aggregation agents such as $\mathrm{NaCl}$ in place of citrate no control over the plasmon shifts could be achieved.

25 Another notable feature of the spectra in Fig. 1 is that the systematic shift in the longitudinal plasmon peak suggests the average cluster size is increasing uniformly throughout the bulk solution rather than creating a very large distribution of cluster sizes. In contrast, end-to-end assembly through 30 selective chemical functionalization of the nanorod end faces results in a rapid broadening and red-shifting of the longitudinal plasmon band with minimal change in the transverse band. ${ }^{8}$ A theoretical rationalisation for the observation of a blue or red-shifted response associated with 35 side-by-side or end-to-end assembly respectively has recently been provided by El-Sayed et $a l^{7}$ however an in-depth understanding of corresponding trends in cluster size, plasmon shift and electric field distribution has yet to be achieved.

An important factor in controlling the assembly process is 40 the bulk concentration of CTAB in the nanorod solution, which was fixed at $1 \mathrm{mM}$. At lower concentrations $(<0.5$ $\mathrm{mM}$; below the cmc value of $0.85 \mathrm{mM}$ ), and with the nanorod concentration unchanged, the assembly process is less well controlled with larger blue shifts difficult to achieve before a 45 final collapse of the colloid suspension. This is due to partial desorption of the CTAB bilayer reducing the surface charge density and the colloidal stability. High fractional surface coverage's of dye adsorbed onto the rod surface can also affect the rod assembly and in our experiments this was kept 50 at a level where a controlled shift was observed as expected for a particular citrate : rod ratio and also produced a good SERRS signal.

In order to create clusters whose plasmon resonance closely matches a targeted excitation laser wavelength it is essential 55 that the assembly process can be quickly halted and the formed clusters stabilised against further aggregation.

The use of polyelectrolytes for the layered electrostatic coating of individual nanorods has been reported by several groups as an important functionalisation step as well as ${ }_{60}$ effectively masking the cytotoxic CTAB layer. ${ }^{15}$ Here, we found that the introduction of a solution of poly-(sodium 4styrenesulfonate), PSS, could be used to halt the aggregation process and thereby stop any further changes in the UV-vis spectra at both low and higher levels of aggregation. The 65 coating process itself also did not induce further aggregation (see Fig. S3, ESI ${ }^{\dagger}$ ). Furthermore, after centrifugation and subsequent resuspension of the stabilised clusters in water, no significant change in the UV-vis spectral profile was observed for over two months (see Fig. S4, $\mathrm{ESI}^{\dagger}$ ).

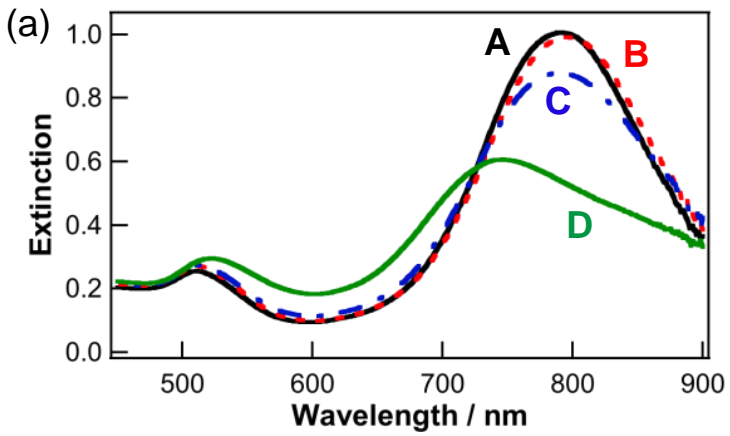

(b)

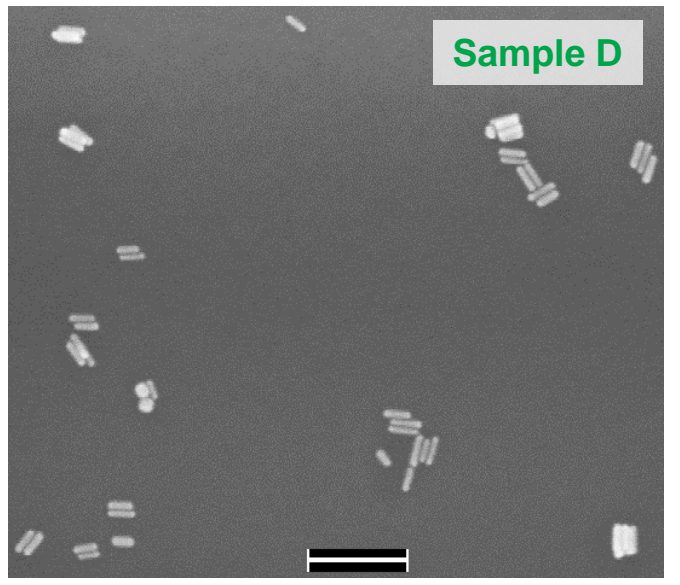

Fig. 2 UV-vis spectra of four PSS-wrapped samples A, B, C and D. Differences between each sample are described in the main text and $\mathrm{ESI}^{\dagger}$. (b) Representative SEM image of sample D (scale bar $=200 \mathrm{~nm}$ ).

Having established the key stages for nanorod assembly 75 and stabilisation, we created a series of samples for characterisation. Fig. 2 displays the UV-vis spectra for 4 different samples all prepared using the same starting volume of stock solution. In addition, a CTAB/DTTCI mixed layer was formed on each sample, except $\mathbf{A}$, prior to aggregation 80 (see $\mathrm{ESI}^{\dagger}$ for details). Both samples A and $\mathbf{B}$ were PSS coated without citrate-induced aggregation while for samples $\mathbf{C}$ and 
D, different concentrations of citrate were added prior to PSS. As expected, the spectra of both samples $\mathbf{A}$ and $\mathbf{B}$ are very similar. Typically, a small blue shift in the longitudinal resonance is observed upon dye adsorption that is associated 5 with coupling between the molecular and plasmon resonances $^{16}$ with a small red-shift occurring upon PSS coating. The longitudinal resonance for sample $\mathbf{C}$ is slightly damped and blue-shifted by $\sim 10 \mathrm{~nm}$ due to a low level of aggregation with a larger shift of $\sim 54 \mathrm{~nm}$ observed for sample ${ }_{10} \mathbf{D}$ where a greater amount of citrate was added.

Fig. 2(b) shows a representative SEM image of sample D where the controlled formation of discrete clusters of aligned nanorods can be clearly observed. Additional images are also provided in the $\mathrm{ESI}^{\dagger}$ for both control (sample B, Fig. S5) and 15 assembled nanorod solutions (samples $\mathbf{C}$ and D, Figs. S6 and S7 respectively). When depositing each sample onto surfacemodified Si substrates for analysis, efforts were made to design a methodology that ensured that the cluster distribution was representative of the bulk solution rather than due to 20 drying-induced aggregation. Measurement of the extent of the nanorod assembly for sample D indicated $21.8 \%$ of each particle/clusters were monomers, $35.3 \%$ dimers and $42.5 \%$ were clusters 3-5 nanorods in size (over 500 discrete particle/clusters counted) with an insignificant amount of 25 larger assemblies observed. This indicates that the vast majority $(>90 \%)$ of the original rod population forms part of an aggregate. For sample $\mathbf{C}$, where less citrate was added, the corresponding fractions were $28.0 \%, 46.2 \%$ and $24.5 \%$ for monomers, dimers and 3-5 rod clusters respectively. In the 30 control samples $\mathbf{A}$ and $\mathbf{B}$, where no citrate was added, no assembly was observed.

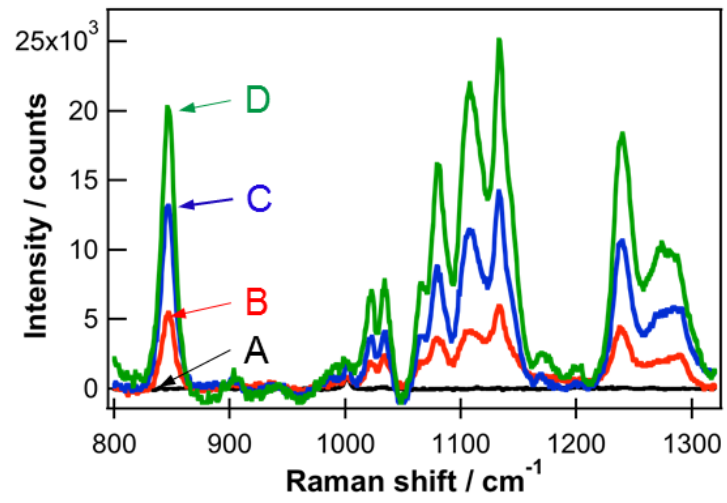

Fig. 3 Ensemble SERRS spectra of samples A, B, C, and D using an excitation wavelength of $785 \mathrm{~nm}$ and accumulation time of $10 \mathrm{~s}$.

35 The corresponding ensemble SERRS spectra obtained for samples A-D are shown in Fig. 3. Sample A has no DTTCI present to prove that $\mathrm{CTAB}$ and the rods do not have a significant contribution to the measured Raman signal, even when aggregated (data for latter not shown). In the presence 40 of DTTCI a large SERRS signal was obtained. Samples B-D also differ in how strongly the plasmon resonance of the cluster overlaps with the $785 \mathrm{~nm}$ laser as well as the solution particle density. Using the SEM results to estimate relative changes in particle concentration combined wih the increased 45 intensity suggests the average SERRS signal per particle is enhanced more than 10-fold for sample $\mathbf{D}$ compared to $\mathbf{B}$.
In summary, we have demonstrated a novel methodology for the solution-based assembly of gold nanorods to form SERRS-active nanotags. The ability to control the side-by50 side alignment and the overall cluster size to form predominantly dimers and trimers as well as the placement of dye reporter molecules between assembled rods offers several advantages compared to spheres for the design of Ramanactive substrates. Also, the stabilising polyelectrolyte layer 55 provides a starting platform for biomolecular functionalization via either electrostatic or covalent attachment ${ }^{17}$ and can also be used to template the growth of thin encapsulating silica layers. ${ }^{18}$ Subsequent work will focus on creating a range of nanomaterials for imaging ${ }^{19}$ and sensing ${ }^{20}$ applications.

60 This work was supported by an Engineering Physical Sciences Research Council (EPSRC) Science and Innovation Award and an EPSRC First Grant award (\#EP/H030468/1).

\section{Notes and references}

$65{ }^{a}$ Centre for Molecular Nanometrology, WestCHEM, Department of Pure and Applied Chemistry, University of Strathclyde, Glasgow, UK, G1 1XL E-mail:alastair.wark@strath.ac.uk Tel: +44 (0)141 5483084

$\dagger$ Electronic Supplementary Information (ESI) available: experimental details and supporting fluorescence, SEM and cluster stability 70 measurements. See DOI: 10.1039/b000000x/

1. C. J. Murphy, A. M. Gole, S. E. Hunyadi, J. W. Stone, P. N. Sisco, A. Alkilany, B. E. Kinard and P. Hankins, Chem. Commun., 2008, 544557.

2. X. Huang, S. Neretina and M. A. El-Sayed, Adv. Mater., 2009, 21, $75 \quad 4880-4910$.

3. G. von Maltzahn, A. Centrone, J.-H. Park, R. Ramanathan, M. J. Sailor, T. A. Hatton and S. N. Bhatia, Adv. Mater., 2009, 21, 31753180.

4. D. Graham and K. Faulds, Chem. Soc. Rev., 2008, 37, 1042-1051.

80 5. H.-S. Park, A. Agarwal, N. A. Kotov and O. D. Lavrentovich, Langmuir, 2008, 24, 13833-13837.

6. N. Zhao, K. Liu, J. Greener, Z. Nie and E. Kumacheva, Nano Lett., 2009, 9, 3077-3081.

7. P. K. Jain, S. Eustis and M. A. El-Sayed, J. Phys. Chem. B, 2006, 85 110, 18243-18253.

8. L. Wang, Y. Zhu, L. Xu, W. Chen, H. Kuang, L. Liu, A. Agarwal, C. Xu and N. A. Kotov, Angew. Chem., Int. Ed., 2010, 49, 5472-5475.

9. K. L. Wustholz, A.-I. Henry, J. M. McMahon, R. G. Freeman, N. Valley, M. E. Piotti, M. J. Natan, G. C. Schatz and R. P. Van Duyne, J. Am. Chem. Soc., 2010, 132, 10903-10910.

10. L. C. Martin, I. A. Larmour, K. Faulds and D. Graham, Chem Commun., 2010, 46, 5247-5249.

11. X. Su, J. Zhang, L. Sun, T.-W. Koo, S. Chan, N. Sundararajan, M. Yamakawa and A. A. Berlin, Nano Lett., 2005, 5, 49-54.

95 12. I. A. Larmour, K. Faulds and D. Graham, J. Phys. Chem. C, 2010, 114, 13249-13254.

13. T. K. Sau and C. J. Murphy, Langmuir, 2004, 20, 6414-6420.

14. B. Nikoobakht and M. A. El-Sayed, Chem. Mater., 2003, 15, $1957-$ 1962.

100 15. A. P. Leonov, J. Zheng, J. D. Clogston, S. T. Stern, A. K. Patri and A. Wei, ACS Nano, 2008, 2, 2481-2488.

16. W. Ni, Z. Yang, H. Chen, L. Li and J. Wang, J. Am. Chem. Soc., 2008, 130, 6692-6693.

17. H. Ding, K.-T. Yong, I. Roy, H. E. Pudavar, W. C. Law, E. J. Bergey and P. N. Prasad, J. Phys. Chem. C, 2007, 111, 12552-12557.

18. A. Guerrero-Martinez, J. Perez-Juste and L. M. Liz-Marzan, $A d v$. Mater., 2009, 22, 1182-1195.

19. A. W. Wark, R. J. Stokes, S. B. Darby, W. E. Smith and D. Graham, J. Phys. Chem. C, 2010, 114, 18115-18120.

110 20. H. R. Sim, A. W. Wark and H. J. Lee, Analyst, 2010, 135, 2528 2532. 
